FLUX

$n^{\circ} 29$

Juillet - Septembre

1997

pp. 1424

\section{Morphogénèse des transports en commun de surface en banlieue parisienne : les occasions manquées du redéploiement ${ }^{1}$}

AGNÈS SANDER

$\mathrm{P}$ OUR QUI S'INTÉRESSE à l'évolution des réseaux techniques dans leurs relations aux territoires, il est assez frappant de constater la très forte permanence de certaines configurations dans la durée, alors que les usages, les fonctionnalités, les techniques, ont souvent subi d'importantes transformations. Le cas du réseau des autobus circulant dans l'Est parisien ${ }^{2}$, auquel nous nous intéressons ici, en est assez exemplaire : la figure actuelle de ce réseau est déjà présente, dans son principe, dans celle prise, lors de leur toute première implantation au cours du XIXe siècle, par les premières lignes de transport en commun de surface implantées hors de Paris (voir Figure 1).

En dehors de toute transformation radicale, le réseau se constitue, par itérations successives, à partir de ces premières lignes. Dans ce cadre, ramifications, prolongations et adjonctions répondent souvent à des demandes fragmentaires de dessertes particulières. Ces modifications successives peuvent toutefois être lues, a posteriori, comme les éléments d'une évolution cohérente : les fragments de rocades créés entre les lignes primitives qui rayonnaient depuis Paris pour atteindre les communes de la périphérie constituent un maillage de plus en plus fin, qui s'étend peu à peu de la limite immédiate de la capitale vers des communes plus éloignées. Les éléments de ce maillage se constituent également par adjonction de lignes rayonnantes vers certains centres-villes, comme Montreuil, et dans une moindre mesure, Romainville et Rosny-sous-Bois.

Les grandes étapes de l'évolution des usages (promenades à la campagne, puis migrations alternantes, puis diversification des motifs de déplacements) des modes de traction (voitures collectives puis omnibus « à traction animale», tramways «à traction animale » puis électrique, autobus enfin) et de gestion (création ou suppression de monopoles), l'ouverture des gares SNCF (1860-1870) dont certaines deviendront RER un siècle plus tard, la prolongation des lignes de métro hors des limites de la capitale (1830-1840 puis 1870-1885), et même, plus récemment (à partir de 1985), une restructuration volontaire de la part de la RATP ne modifieront qu'à la marge la physionomie d'un réseau constitué au fil de micro-évolutions incrémentales. 


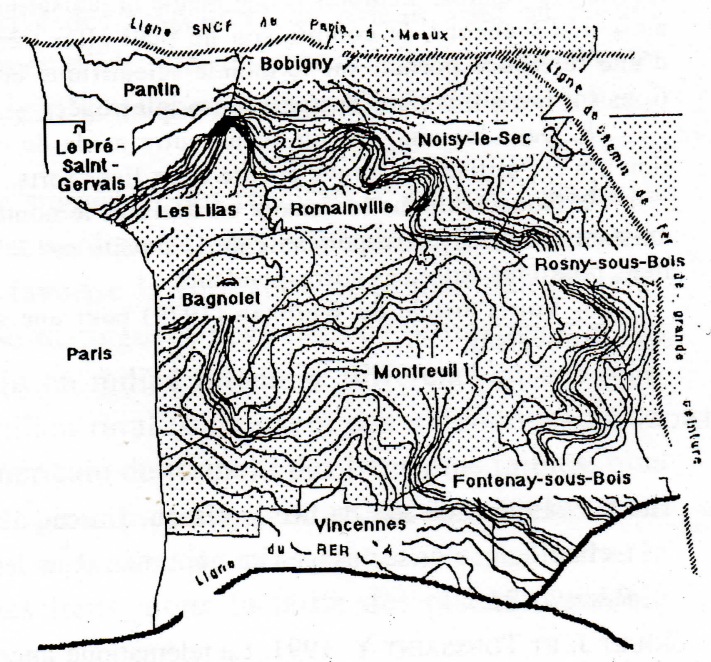

Figure 1 : Le site de l'investigation : limites constituées par Paris et par les lignes de chemin de fer, avec, en grisé, l'emprise du secteur « Est parisien » des récentes restructurations de la RATP (source : Legendre d'Anfray, Sander 1993).

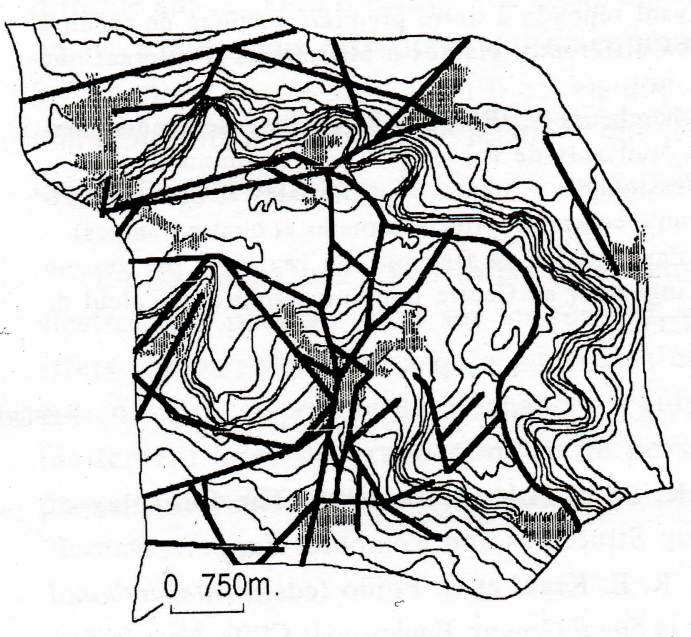

Figure 1 bis : Le site de l'investigation : voies royales et percées « volontaires », avec, en grisé, les secteurs urbanisés en 1867 (sources : Carte des tramways du département de la Seine 1867; Hanning 1975).
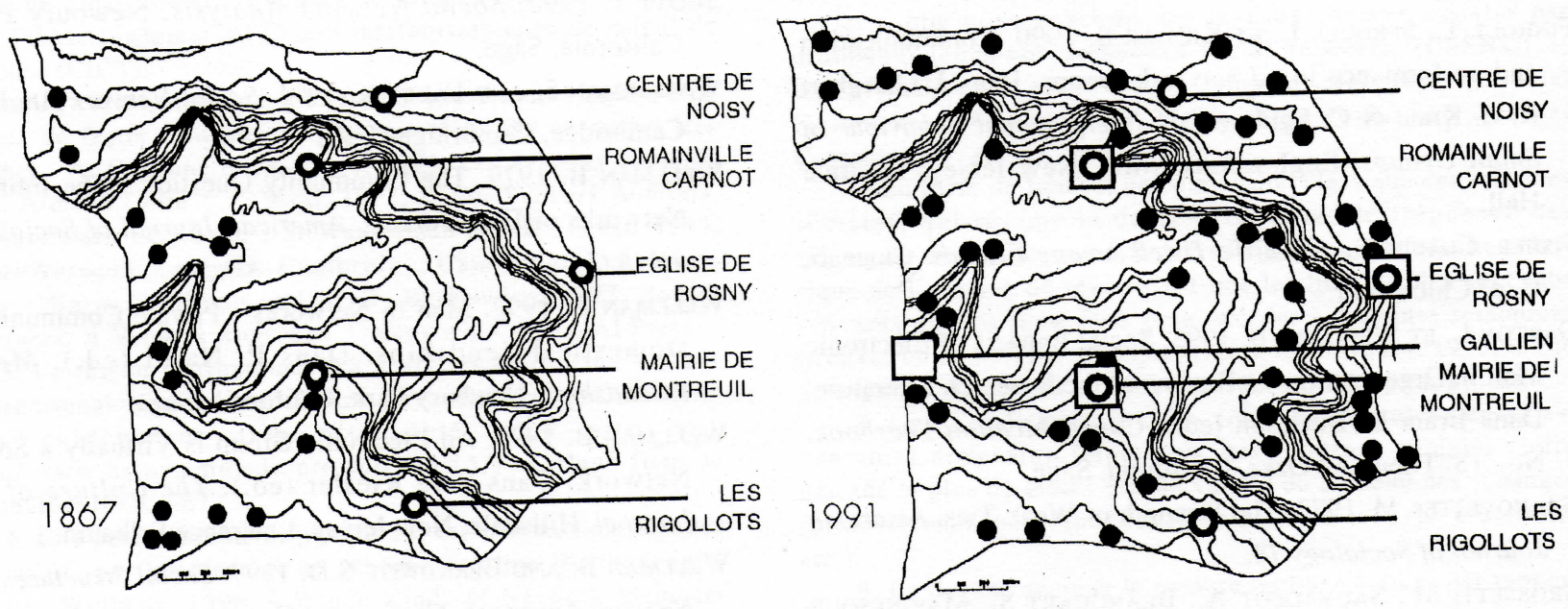

Figures 2 et 2 bis : Localisation des points de correspondance entre autobus en 1867 et 1991. Les principaux points de correspondance de l'époque actuelle étaient déjà présents en 1867 ; l'ensemble des points reste localisé en périphérie du plateau. Les cercles noirs représentent les points de correspondance entre autobus dont la position a varié au fil du temps. Les cercles blancs indiquent ceux dont la position est restée fixe de 1867 à nos jours. Les carrés blancs sont aujourd'hui les points de correspondance principaux, choisis par la RATP pour devenir les « points-clés » de ses restructurations (source : Legendre d'Anfray, Sander, 1993). 
Ces observations peuvent être rapprochées avec profit du modèle de développement des réseaux techniques élaboré par J.-M. Offner (1993) distinguant sept phases de développement (naissance, développement initial, transformation/redéploiement, maturité, déclin, disparition), modèle que l'auteur propose d'utiliser non seulement pour l'analyse, mais également de manière prospective. Nous nous interrogeons donc, en conclusion, sur la capacité des autobus à trouver un «second équilibre » (phase de redéploiement) qui paraît n'avoir toujours pas été atteint aujourd'hui, alors qu'il s'agit de l'une des phases-clés de la croissance, intensive ou extensive, d'un réseau technique ; nous nous demandons également si l'autobus, trop fortement concurrencé par d'autres modes, ne risque pas d'atteindre une phase de déclin, en l'absence de décisions qui affirmeraient nettement sa complémentarité avec l'ensemble du système des transports de la Région parisienne.

\section{LE DÉVELOPPEMENT INITIAL : UNE ÉTAPE DÉTERMINANTE}

La thèse de la phase de développement initial comme prépondérante dans la constitution des réseaux de transport en région parisienne a été développée par Nicholas Papayanis (Papayanis 1993) dans les domaines de l'organisation et de la gestion. Pour lui, « la toute première formation du système public des transports a été plus révolutionnaire que sa mécanisation ultérieure, et la formation de la $\mathrm{CGO}$ et de la $\mathrm{CIV}^{3}$ ont constitué l'acte final de la création du système circulatoire du Second Empire à Paris ». Cette première étape fige une organisation centralisée du système des transports dans ses relations à la croissance urbaine et à une «géographie » sociale de la capitale. Nos travaux personnels, fondés sur une analyse d'abord cartographique de l'évolution du tracé des lignes, mettent en évidence des phénomènes similaires, lorsqu'il s'agit de la topologie du réseau, envisagée dans sa relation avec la topographie et l'urbanisation du site d'insertion.

\section{L'IMPORTANCE DE LA CONFIGURATION DU RÉSEAU-SUPPORT}

Dès que les premières lignes de voitures à cheval collectives à faible capacité, destinées essentiellement, semble-t-il, à la promenade, s'installent en région parisienne, elles suivent tout naturellement les routes principales (voies royales ou routes percées du XVIII ${ }^{\mathrm{e}}$ siècle) reliant Paris aux communes périphériques (Figure 1 bis). Les lignes sont exploitées par de très nombreuses entreprises distinctes, et constituent, compte tenu des voies de desserte, des radiales. Peu à peu, ces lignes sont prolongées, toujours selon le même schéma, et le nombre de voitures augmente.

En 1859, une ligne de chemin de fer voyageurs est ouverte de Paris vers la Varenne-Saint-Hilaire (actuel RER A dans sa partie Sud). L'ouverture de la gare de Vincennes sur cette ligne conduit la CGO à restreindre très fortement l'activité de son centre de départ situé à la Bastille (Lagarrigue 1956) ${ }^{4}$. Sur les cartes de cette époque, certains prolongements au delà de Vincennes sont en effet supprimés, et le nombre de lignes vers cette commune diminue. C'est au même moment qu'apparaissent de premiers éléments de liaison intrabanlieue, par l'intermédiaire de « services rabatteurs ${ }^{5}$.

Lors de cette première ramification du réseau, destinée à relier les communes sans chemin de fer à celles où existe une gare, les routes empruntées restent, compte tenu de la mauvaise qualité des autres voies, celles ouvertes par les ingénieurs des Ponts et Chaussées au cours du XVIIIe siècle (Arbelot 1973 ; Picon 1992 ; Lepetit 1986), les «percées volontaires », dans ce même but de relation intra-communale. Le site retenu pour l'étude en est particulièrement caractéristique, car il est constitué d'un plateau entouré de thalwegs, à l'intérieur desquels se sont installés les villages qui constituent aujourd'hui les centres anciens de ces communes de banlieue (Avramides et al.).

\section{UNE GRANDE PERMANENCE DE LA CONFIGURATION ORIGINELLE}

L'organisation du réseau-support est ainsi déterminante pour la constitution de la structure du réseau-service : le transport collectif « de surface ». Cette organisation est elle-même fonction des premières implantations humaines dans un site très contraint : les voies principales suivent en effet les lignes des thalwegs ou les courbes de niveaux constituant la limite du plateau (Hanning 1975). 
La voirie comme réseau-support gardera son importance jusqu'à nos jours, non seulement en contraignant d'une certaine manière, les lignes d'autobus anciennes à conserver leurs tracés initiaux, mais aussi en autorisant de nouveaux parcours : il est amusant de constater que la ligne de rocade créée à l'occasion des récentes (1991) restructurations du réseau des autobus de la RATP en banlieue parisienne emprunte un chemin de bord de plateau ouvert tardivement (1840-1860 : un siècle après les percées volontaires) pour relier entre eux les forts avancés des fortifications de Paris. Tout se passe comme si chaque voie percée était destinée à recevoir une ligne de transport en commun, mais après une période d'attente d'un siècle au moins.

Une étude menée pour la RATP sur le site dont il est ici question (Legendre d'Anfray, Sander 1993) a également mis en évidence la grande permanence de la localisarion des points de correspondance entre autobus : la position des plus importants d'entre eux (en termes de nombre de lignes aujourd'hui) n'a pas varié depuis 1867. De plus, aucun de ces points n'est situé sur le planeas luii-même : aujourd'hui encore, malgré une extension massive de l'urbanisation depuis un siècle, ils resnent localisés en périphérie (Figures 2 et 2 bis). Ils sont nous, à l'exception de Galliéni, d'implantation plus tardives situés au croisement de percées volontaires, à proximivé des centres-villes anciens, position qui entraîne detleurs aujourd'hui un certain nombre de difficultés diménagement ${ }^{6}$.

Ces difficultés, tout comme l'absence d'évolution du positionnement des points de correspondance, mettent clairement en évidence que la topologie et le tracé du réseau des autobus en banlieue parisienne doivent ėre regardés plus comme les résultats d'évolutions successives, cohérentes a posteriori, que comme les éléments d'une organisation qui aurait été planifiée pour être, à chaque époque successive, en parfaite adéquation avec l'urbanisation du secteur.

\section{LES OCCASIONS MANQUÉES DU REDÉPLOIEMENT}

Comme la chronologie (voir p. 21) le met en évidence, de nombreuses opportunités d'évolution se sont pourtant présentées au réseau des transports en commun de surfa- ce en région parisienne. Elles ont, en général, suscité des modifications marginales de la figure du réseau (Figure 3), qu'il n'est guère possible d'appeler « redéploiement», au sens retenu par J.-M. Offner (1993).

Les cartes évolutives présentées ici mettent en évidence le fait que la figure du réseau des autobus actuel est déjà présente, en germes, en 1925. L'évolution est en réalité très faible entre la carte de 1913 (non représentée ici) et celle de 1925 . On peut donc soutenir que cette figure est constituée, pour l'essentiel, dès avant la première guerre mondiale : les liaisons radiales depuis Paris vers les centres-villes, qui s'installent au début du siècle, sont complétées par une ligne de rocade ; les principaux points de correspondance actuels sont déjà tous constitués par le croisement de deux lignes au moins ; la position «privilégiée » de Mairie de Montreuil commence à apparaître. Celle-ci sera confirmée en 1937, lorsque conjointement avec l'ouverture d'une station de métro, terminus de la ligne 9, sera installée une série importante de lignes de rabattement «en étoile » autour de ce centre. Les années suivantes verront se compléter peu à peu les éléments d'une rocade plus éloignée de Paris, qui sera achevée en 1993 lors de restructurations effectuées par la RATP. On notera, d'autre part, que les intervalles de temps entre les cartes montrées ici étant assez longs, les apparitions/disparitions/réapparitions de certaines dessertes ne sont pas restituées. L'augmentation apparemment constante du nombre des dessertes n'est donc pas aussi linéaire que la représentation graphique le laisse supposer.

\section{UNE ÉVOLUTION DU MATÉRIEL ROULANT ET DES MODES DE TRACTION}

La continuité dans le temps des tracés empruntés par les lignes de transport collectif de surface le montre : les modes successifs empruntent des cheminements similaires. Dans quelques rares cas, des améliorations techniques autorisent de nouvelles dessertes, comme à Bagnolet et aux Lilas, communes difficiles à atteindre en raison d'une trop forte pente, au tout début du XIX siècle. Une nouvelle desserte vers Bagnolet apparaît ainsi en 1856. Celle-ci semble avoir été rendue possible par un système de freins améliorés (Daumas et al. 1977). En règle générale, tout se passe pourtant comme si les modes se succédaient sans que jamais les tracés ne 
soient remis en question. Le remplacement des tramways par les autobus en est particulièrement typique.

Au début du XIX ${ }^{\mathrm{e}}$ siècle, les véhicules utilisés sont des «voitures publiques » de Paris à destination des environs. Celles-ci, de capacité insuffisante, seront peu à peu remplacées par des omnibus (traction à cheval, capacité plus importante que les voitures collectives), puis par les tramways (traction à cheval puis électrique), lesquels céderont à leur tour la place aux autobus (moteurs à essence), pour retrouver, très récemment, une certaine popularité.

En 1856, un premier tramway apparaît en banlieue parisienne, à l'Ouest de la capitale. Trois lignes de tramways y circulent en 1867 . Ce nombre atteint en 1879 la valeur de 29 , pour l'ensemble de la banlieue. Il est à noter que la CGO refusant toute amélioration technique de ses véhicules, l'Etat et le Conseil Municipal de Paris réussissent, dans la perspective de l'exposition universelle de 1900 et grâce à une loi votée en 1880, à contourner le monopole de la CGO en concédant, le 5 juin 1891, certaines lignes de tramway (à traction électrique) à des compagnies exploitant des pénétrantes vers la capitale. Ce mode présente en effet l'intérêt, grâce à la diminution des frottements des roues sur les rails, de pouvoir offrir une capacité supérieure, à traction égale. À partir de 1902, toutes les dessertes sont assurées par des tramways, à l'exception de quelques voitures publiques à « itinéraires et horaires facultatifs » (Lagarrigue 1956).

Compte tenu de ce développement rapide et massif des tramways, leur disparition en banlieue semble s'expliquer par un effet «d'entraînement » du modèle parisien (Offner 1988). En effet, les conditions d'exploitation étaient bien plus satisfaisantes en banlieue que dans la capitale, où plusieurs facteurs se conjuguent pour conduire à leur déclin. Il aurait pourtant été possible d'envisager une complémentarité entre les deux modes, le tramway utilisant les voies les plus importantes, fixant ainsi une infrastructure de desserte rapide à la grande capacité, alors que l'autobus, réputé plus souple, aurait assuré des dessertes plus fines et évolutives (Daumas et al. 1977)7

De fait, la disparition des tramways sur le site étudié ici ne suit pas le modèle, à l'œuvre pour d'autres réseaux (Offner 1993), de complémentarité d'abord, concurrence ensuite : les autobus se substituent au mode ancien sur les dessertes entre Paris et les communes les plus proches, pour s'étendre très vite au-delà, selon des étapes assez similaires à celles de l'implantation des toutes premières voitures collectives. Les autobus commencent ainsi à s'étendre vers la banlieue en 1921 (17 lignes pour l'ensemble de la banlieue en 1925), et les premiers apparaissent dans notre secteur vers 1930. Une carte de la STCRP (Société des Transports en Commun de la Région Parisienne), datée de 1931, permet de distinguer les tracés empruntés par les autobus de ceux suivis par les tramways. Cette carte est la seule que nous ayons retrouvée entre le «tout tramway » (1925) et le «tout autobus» $(1938)^{8}$. Les lignes d'autobus présentes dans l'Est parisien à cette date semblent se superposer aux tramways - ou les remplacer - sur certains grands itinéraires à destination des centresvilles les plus proches de Paris (Pantin, Les Lilas, Bagnolet, Montreuil) et assurer d'autre part une desserte un peu plus fine qu'en 1925 dans le centre de Vincennes, en fin de trajet. Les «prolongations» audelà de ces premières communes sont toujours assurées par des tramways, et ne seront remplacées par des autobus que dans une seconde phase.

C'est ainsi que lorsque la Société des Transports en Commun de la Région Parisienne (créée en 1920) procède dans un premier temps à une modernisation du matériel, puis dans un second à la suppression des tramways, la figure du réseau n'en subit pas de modifications, seule la capacité des véhicules s'accroît.

\section{DES ÉVOLUTIONS INSTITUTIONNELLES}

Compte tenu de la faible quantité de dessertes en banlieue et de leur passage quasi-obligé par les voies les plus importantes, le nombre de compagnies gestionnaires et leur organisation semble peu influer sur la figure du réseau de surface.

Dans une première phase d'extension au-delà des communes les plus proches de la capitale, la grande diversité des entreprises conduit à dédoubler le nombre des véhicules atteignant cette première ceinture, une compagnie proposant une desserte de Paris vers ces petites villes et une autre, offrant de relier Paris à une commune plus lointaine, assurant un arrêt dans la commune intermédiaire. 

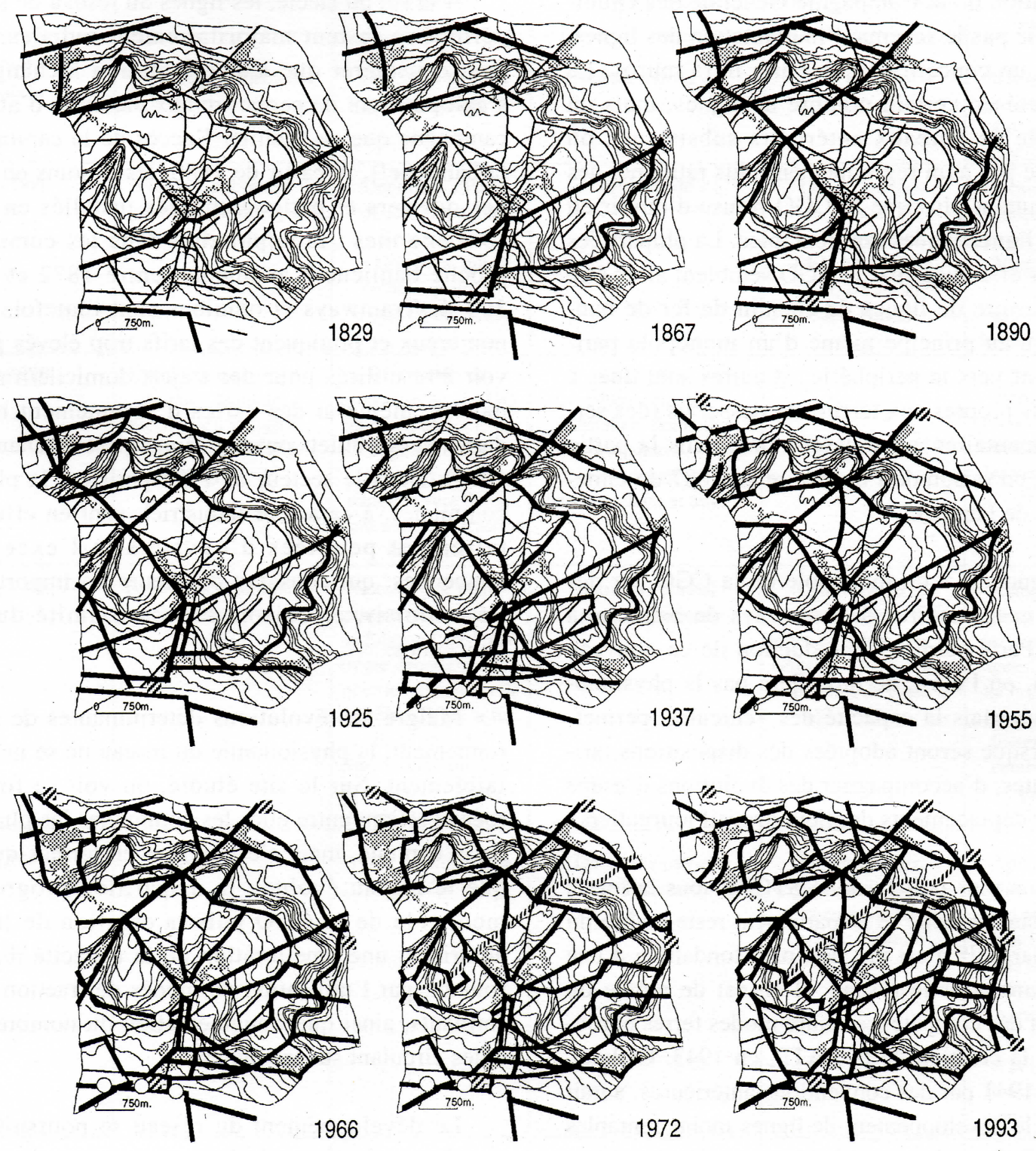

\section{Légende des cartes :}

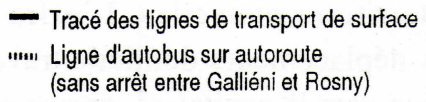

\% Gare SNCF voyageur ou RER RATP

Station de métro

- percée "volontaire"

Courbes de niveaux

Zones urbanisées en 1867

Figure 3 : Évolution de la figure du réseau des transports collectifs de surface.

Les cartes évolutives présentées ici mettent en évidence le fait que la figure du réseau des autobus actuel est déjà présente, en germes, en 1925. L'évolution est en réalité très faible entre la carte de 1913 (non représentée ici) et celle de 1925. On peut donc soutenir que cette figure est constituée, pour l'essentiel, dès avant la première guerre mondiale : les liaisons radiales depuis Paris vers les centres-villes, qui s'installent au début du siècle, sont complétées par une ligne de rocade ; les principaux points de correspondance actuels sont déjà tous constitués par le croisement de deux lignes au moins ; la position "privilégiée » de Mairie de Montreuil commence à apparaître. Celle-ci sera confirmée en 1937, lorsque conjointement avec l'ouverture d'une station de métro, terminus de la ligne 9, sera installée une série importante de lignes de rabattement « en étoile » autour de ce centre. Les années suivantes verront se compléter peu à peu les éléments d'une rocade plus éloignée de Paris, qui sera achevée en 1993 lors de restructurations effectuées par la RATP. On notera, d'autre part, que les intervalles de temps entre les cartes montrées ici étant assez longs, les apparitions / disparitions / réapparitions de certaines dessertes ne sont pas restituées. L'augmentation apparemment constante du nombre des dessertes n'est donc pas aussi linéaire que la représentation graphique le laisse supposer. 
La formation de la Compagnie Générale des Omnibus ne modifie pas le schéma d'implantation des lignes, le monopole ne concernant que Paris intra-muros. La CGO a racheté un certain nombre de lignes, mais un grand nombre de bureaux antérieurs subsistent : on n'assiste donc pas à un regroupement plus rationnel des dessertes, d'autant plus que la CGO refuse d'ouvrir en banlieue des lignes jugées non rentables. La plupart des modifications effectuées par la CGO semblent alors être liées à l'ouverture de lignes de chemin de fer de banlieue 9 plus qu'au principe même d'un monopole parisien s'étendant vers la périphérie ; d'autres sont dues à des évolutions propres aux territoires desservis (des services supplémentaires apparaissent ainsi dans la partie Nord du site, en raison de l'ouverture en 1887 du cimetière parisien de Pantin).

Le « grignotage » du monopole de la CGO par des compagnies gestionnaires de tramways de banlieue à direction de Paris permet l'introduction de ce nouveau mode, ce qui, on l'a vu, ne modifiera pas la physionomie du réseau, mais la capacité des véhicules, permettant ainsi, lorsque seront adoptées des dispositions tarifaires adéquates, d'accompagner des évolutions d'usage induisant des déplacements domicile/travail journaliers.

La création plus tardive de la STCRP sous la forme d'une régie dans laquelle le Département reste maître du cahier des charges ${ }^{10}$ n'est pas non plus fondatrice d'une nouvelle figure pour le réseau. Il en est de même du regroupement des compétences entre modes ferrés et routiers, lors de la création de la RATP, en 1943. Celle-ci, préparée dès 1941 par des conventions antérieures, aurait pu permettre le développement de lignes moins rentables par équilibrage financier entre ferré et routier. Cet objectif ne sera toutefois atteint que très partiellement.

\section{DES ÉVOLUTIONS DES USAGES ET DE L'URBANISATION EN PÉRIPHÉRIE}

Au début du XIX ${ }^{\mathrm{e}}$ siècle, les premières «voitures publiques » semblent avoir pour but essentiel la promenade. Il en est de même de la ligne de chemin de fer desservant à partir de 1859 La Varenne-Saint-Hilaire, qui aurait été utilisée surtout pour des promenades à Vincennes et au bord de la Marne.
À la fin du siècle, les lignes du réseau de surface en périphérie restent majoritairement radiales, mais on peut considérer que leur orientation a changé : il ne s'agit plus tant de permettre aux parisiens d'atteindre la campagne que de faciliter l'accès de la capitale depuis la banlieue ${ }^{11}$. À partir de 1880, les besoins en transport des ouvriers et petits employés installés en banlieue sont énormes : la population dans les communes de proche banlieue a augmenté, entre 1872 et 1911, de $300 \%$. Tramways et omnibus sont toutefois trop peu nombreux et pratiquent des tarifs trop élevés pour pouvoir être utilisés pour des trajets domicile/travail journaliers, même si des cartes d'abonnement hebdomadaires et des billets ouvriers apparaissent (Daumas et al. 1977). Dans le secteur de l'Est parisien, la plupart des communes, à caractère industriel, sont en effet majoritairement peuplées d'ouvriers, à l'exception de Vincennes, qui conserve d'ailleurs un important trafic lié au loisir, en raison de la proximité du bois de Vincennes.

Malgré ces évolutions déterminantes de son environnement, la physionomie du réseau ne se modifie que faiblement. Sur le site étudié, on voit se former une rocade reliant entre elles les communes les plus proches de la capitale, mais c'est surtout en termes quantitatifs que le réseau évolue : la disparition progressive du monopole de la CGO puis la création de la STCRP autorisent une amélioration de la capacité du matériel roulant, par l'utilisation de modes de traction plus performants, ainsi que l'augmentation du nombre de véhicules circulant sur les lignes.

Le développement du réseau se poursuit alors de manière extensive après la seconde guerre mondiale, en se densifiant peu à peu pour répondre à des demandes de desserte du territoire nouvelles. L'offre privilégie massivement les déplacements domicile/travail journaliers de la banlieue vers la capitale ${ }^{12}$, que se soit par des lignes radiales ou par rabattement vers les stations des modes lourds.

À partir des années 1980, un certain nombre d'évolutions structurelles conduisent les opérateurs de transport collectif à envisager des modifications de leur offre. La première évolution est celle de l'augmentation des déplacements en automobile, face à laquelle le transport en commun doit s'adapter. Sa structure doit rendre possible des parcours en rocade; des chaînes de 


\section{Sander - Morphogénèse des transports en commun de surface en banlieue parisienne}

\begin{tabular}{|c|c|c|c|c|c|}
\hline Période & Transport de surface & Opérateur & Usages & Autres modes sur le site & $\begin{array}{c}\text { Évolution du réseau (voir } \\
\text { aussi la figure 3) }\end{array}$ \\
\hline $\begin{array}{l}\text { Première moitié du } \\
\mathrm{XIX}^{\mathrm{e}} \text { siècle }\end{array}$ & $\begin{array}{l}\text { Voitures publiques de } \\
\text { Paris vers les environs }\end{array}$ & $\begin{array}{l}\text { Multiples petites } \\
\text { compagnies }\end{array}$ & «Promenade » & & $\begin{array}{l}\text { Radiales au départ } \\
\text { de Paris, peu à peu } \\
\text { prolongées au delà } \\
\text { des communes les } \\
\end{array}$ \\
\hline 1855 à 1880 & $\begin{array}{l}\text { Voitures publiques, } \\
\text { omnibus, tramways } \\
\text { (traction animale) }\end{array}$ & $\begin{array}{c}\text { Monopole à Paris } \\
\text { de la Compagnie } \\
\text { Générale des } \\
\text { Omnibus, qui } \\
\text { rachète quelques } \\
\text { lignes en banlieue. }\end{array}$ & «Promenade » & $\begin{array}{l}\text { Ouverture d'un } \\
\text { chemin de fer } \\
\text { voyageur (futur } \\
\text { RER A) de Paris } \\
\text { vers La-Varenne- } \\
\text { Saint-Hilaire }\end{array}$ & $\begin{array}{l}\text { Premiers éléments de } \\
\text { liaisons inter-ban- } \\
\text { lieues vers la gare de } \\
\text { Vincennes ; dispa- } \\
\text { ritions de quelquès } \\
\text { lignes de surfaces sur } \\
\text { le tracé du mode ferré }\end{array}$ \\
\hline 1880 à 1920 & $\begin{array}{l}\text { Omnibus, tramways } \\
\text { (traction animale, } \\
\text { électrique), voitures } \\
\text { publiques. Dès } 1902, \\
\text { tramways seuls }\end{array}$ & \begin{tabular}{|c} 
Restriction du \\
monopole de la CGO \\
au profit de sociétés \\
exploitant des \\
tramways de la \\
banlieue vers Paris, \\
ayant adopté la traction \\
électrique \\
\end{tabular} & $\begin{array}{c}\text { Début des } \\
\text { déplacements } \\
\text { journaliers domicile/ } \\
\text { travail avec } \\
\text { abonnements } \\
\text { hebdomadaire et billets } \\
\text { ouvriers }\end{array}$ & & $\begin{array}{l}\text { Formation d'une } \\
\text { rocade reliant entre } \\
\text { elles les communes } \\
\text { les plus proches de } \\
\text { la capitale }\end{array}$ \\
\hline 1920 à 1948 & $\begin{array}{c}\text { Tramways et autobus, } \\
\text { autobus seuls à partir } \\
\text { de } 1938\end{array}$ & \begin{tabular}{|c|} 
Société des Trans- \\
ports en Commun de \\
la Région Parisienne \\
(régie départementale \\
pour les transports de \\
surface). Vers la fin \\
de la période, \\
conventions amorçant \\
la fusion des réseaux \\
ferrés et de surface. \\
\end{tabular} & $\begin{array}{l}\text { Domicile/ travail } \\
\text { essentiellement }\end{array}$ & $\begin{array}{c}\text { À partir de 1933, } \\
\text { prolongements des } \\
\text { lignes de métro vers les } \\
\text { centres-villes les plus } \\
\text { proches de Paris }\end{array}$ & $\begin{array}{c}\text { Remplacement pro- } \\
\text { gressif des tramways } \\
\text { par des autobus sur les } \\
\text { lignes les plus proches } \\
\text { de la capitale puis sur } \\
\text { les plus éloignées. La } \\
\text { desserte bus dans les } \\
\text { centres-villes est un } \\
\text { peu plus fine } \\
\text { qu'auparavant }\end{array}$ \\
\hline 1948 à 1970 & Autobus & $\begin{array}{l}\text { 1948: création de la } \\
\text { RATP }\end{array}$ & $\begin{array}{c}\text { Domicile/ travail } \\
\text { essentiellement }\end{array}$ & $\begin{array}{c}\text { Ouverture aux } \\
\text { voyageurs de la ligne } \\
\text { de chemin de fer de } \\
\text { grande ceinture }\end{array}$ & $\begin{array}{l}\text { Après la guerre, un } \\
\text { plan de restructura- } \\
\text { tion prévoit le rabat- } \\
\text { tement des autobus } \\
\text { vers les terminus du } \\
\text { métro. Seul élément } \\
\text { subsistant } 10 \text { ans } \\
\text { après : suppression de } \\
\text { la ligne de bus } \\
\text { doublant le trajet du } \\
\text { métro vers Montreuil }\end{array}$ \\
\hline 1970 à 1984 & Autobus & $\begin{array}{c}\text { RATP (autres } \\
\text { transporteurs en limite } \\
\text { du secteur) }\end{array}$ & $\begin{array}{c}\text { Assimilé par } \\
\text { l'opérateur à domicile/ } \\
\text { travail }\end{array}$ & $\begin{array}{l}\text { Prolongement du } \\
\text { métro vers Galliéni, } \\
\text { (1971), ouverture de } \\
\text { deux gares de grande } \\
\text { ceinture, en inter- } \\
\text { connexion avec le } \\
\text { RER A (1980) }\end{array}$ & $\begin{array}{l}\text { Développement } \\
\text { extensif du réseau }\end{array}$ \\
\hline 1984 à 1990 & $\begin{array}{l}\text { Autobus en limite du } \\
\text { secteur, tramway } \\
\text { Saint-Denis-Bobigny }\end{array}$ & $\begin{array}{c}\text { RATP (autres } \\
\text { transporteurs en limite } \\
\text { du secteur) }\end{array}$ & $\begin{array}{l}\text { Reconnaissance de la } \\
\text { diversification des } \\
\text { usages, de } \\
\text { déplacements « en } \\
\text { réseaux », de } \\
\text { l'augmentation des } \\
\text { déplacements } \\
\text { interbanlieue }\end{array}$ & $\begin{array}{l}\text { Prolongement du } \\
\text { métro vers } \\
\text { Bobigny-Pantin- } \\
\text { Raymond Queneau } \\
\text { (1985) }\end{array}$ & $\begin{array}{c}\text { Opérations de } \\
\text { restructurations } \\
\text { «Autrement Bus » en } \\
\text { banlieue. Focalisation } \\
\text { sur les points de } \\
\text { correspondance, } \\
\text { apparition d'une } \\
\text { rocade en limite } \\
\text { extérieure du site }\end{array}$ \\
\hline
\end{tabular}


déplacement, à repenser selon les polarisations de l'espace, doivent pouvoir être pratiquées sans que les ruptures de charge ne soient trop pénibles, et ce quelque soit l'ensemble des modes successivement utilisés; le confort, enfin, doit être amélioré en station tout autant que dans les véhicules. Comme l'écrit un chercheur de l'Unité Prospective de la RATP, « le produit transport ne peut plus [...] être défini comme une simple circulation de flux mais doit être intégré dans une notion plus vaste qui est celle de la mobilité urbaine» (Peny 1990).

C'est dans ce contexte que la RATP lance, à partir de 1989, des opérations de restructuration de son réseau d'autobus en banlieue parisienne. La banlieue est alors divisée en sept secteurs d'interventions, sur lesquels pourront être concentrées de nombreuses d'innovation. Il s'agit essentiellement, afin de privilégier la vision du voyageur, de faciliter la correspondance entre autobus et d'améliorer la lisibilité du réseau, de simplifier et de hiérarchiser celui-ci en mettant en avant la volonté de fabriquer un réseau «par points» (et non plus par lignes). Tous les objectifs ne seront pas atteints, et les opérations Autrement Bus se concrétiseront essentiellement par la mise en place de «points-clés », ces lieux de la correspondance entre autobus à l'architecture spécifique, où se croisent de nombreuses lignes ; par la production d'une cartographie et d'une signalétique améliorant notamment lisibilité et repérage et par une évolution des missions confiées aux dépôts.

Sur le site de cette étude, les quatre points-clés finalement retenus sont Mairie de Montreuil, RomainvilleCarnot, Eglise de Rosny et Galliéni. À l'exception de Galliéni, d'implantation plus tardive, il était possible de repérer ces points comme lieux singuliers du territoire (croisement de percées volontaires puis des premières lignes de transport de surface) dès le XIX ${ }^{e}$ siècle. L'opération «Autrement Bus » menée dans l'Est parisien aura donc confirmé des tendances préexistantes plus que remis en cause la structure du réseau. Elle aura, en outre, amélioré l'intermodalité à Rosny-Bois-Perrier et Val-de-Fontenay et implanté une ligne en rocade en limite du plateau.

\section{L'INTRODUCTION DES MODES FERRES}

En juillet 1928, quinze prolongements de lignes de métro sont déclarés d'utilité publique en raison du développement démographique et de l'accroissement des échanges entre Paris et les communes limitrophes. Sept sont réalisés entre 1934 et 1937 , dont quatre dans le site étudié ici : l'ouverture, en 1934, du tronçon Porte de Vincennes-Château de Vincennes a renforcé le point d'échange entre autobus qui existait initialement à la station château de Vincennes. La ligne 9 (Porte de Montreuil) a été prolongée entre 1933 et 1937. La ligne 11 a été prolongée jusqu'à Mairie des Lilas en 1937, mais l'ouverture d'une station à Romainville-Carnot, prévue en 1928, n'a jamais été réalisée. La ligne 5 est prolongée en 1942, de Place d'Italie à Eglise de Pantin. La ligne de Grande ceinture, quant à elle, est ouverte aux voyageurs entre 1952 et 1954, sans que les tracés des lignes de surface ne se modifient de manière conséquente.

Après la seconde guerre mondiale, un plan pour la remise en service du réseau des autobus est élaboré, qui prévoit de concentrer l'effort sur le rabattement aux terminus de métro, en supprimant les pénétrantes vers Paris. En fait, la carte de 1955 met en évidence la très forte similarité entre le réseau reconstitué après la guerre et celui de 1937 : la seule différence attribuable au plan de restructuration prévu est la suppression de la ligne d'autobus doublant la ligne 9 du métro. Cette opportunité de mise à plat du réseau n'aura donc pas été saisie.

Un prolongement ultérieur du métro a lieu en 1971. L'ouverture de la station Galliéni coïncide avec l'implantation d'une importante gare routière (Margail et al. 1996) et avec le percement de l'autoroute A3, sur laquelle circulent aujourd'hui trois lignes d'autobus (représentées par une ligne grisée sur les cartes) qui vont directement de Galliéni au centre commercial de Rosny 2, pour se prolonger au-delà du secteur étudié. La station de métro la plus récente est Bobigny-PantinRaymond-Queneau (1985), pour laquelle d'importantes études ont été menées sur le thème de l'intermodalité. Peu avant ont été ouvertes les gares de grande ceinture « Rosny-Bois-Perrier » et « Val-de-Fontenay » (1980), cette dernière regroupant aujourd'hui un nombre important de lignes d'autobus en correspondance avec le RER A. 
On ne constate, au total, que peu de modifications du réseau de surface sur le secteur de l'Est parisien qui serait dues à l'introduction des axes lourds.

- Les prolongations des lignes de métro, dans la première moitié du siècle, viennent renforcer les points principaux de correspondance entre autobus préexistants, situés aux terminus des lignes de métro, au détriment des stations de métro intermédiaires (entre la limite de Paris et les terminus), qui ne sont pas considérées comme des points de rabattement. La suppression de la ligne d'autobus « remplacée » par la ligne 9 du métro interdit ainsi toute correspondance entre autobus à la station «Robespierre », située entre la Porte de Paris et Mairie de Montreuil.

- L'ouverture, un siècle auparavant, de la gare de Vincennes n'avait pas non plus généré de modifications dans le tracé des lignes de surface : aujourd'hui, les lignes d'autobus ne desservent toujours pas directement la gare RER de Vincennes, les arrêts les plus proches étant situés à environ une inter-station de la gare proprement dite.

- Le plan de restructuration par rabattement sur les terminus du métro, envisagée après guerre, n'aura pas non plus réussi à s'imposer et seule, finalement, l'opération Autrement Bus aura apporté quelques améliorations dans l'intermodalité par modification du tracé de certaines lignes. Notons toutefois que cette intermodalité concerne uniquement les modes lourds appartenant à la RATP : à Rosny-sous-Bois, par exemple, le «Point-clé » créé lors de l'opération Autrement Bus n'est pas situé près de la gare SNCF de grande ceinture qui dessert la ville, mais dans le centre-ville.

\section{UN RÉSEAU QUI N'A PAS ENCORE CONNU DE TRANSFORMATION MAJEURE}

Les tentatives les plus volontaristes de restructuration du réseau des transports de surface en région parisienne paraissent ainsi vouées à l'échec. La phase de la naissance, très dépendante de la configuration des voiries et du site géographique, est déterminante : elle semble conditionner fortement toute évolution future du réseau. Même les transformations d'usage, qui souvent entrấnent des évolutions profondes des infrastructures et des services, ne semblent jouer qu'un faible rôle dans le redéploiement de l'offre de transport. Une nouvelle étape de transformation par adaptation aux modes de vie métropolitains et de redéploiement par hiérarchisation de l'offre, le bus s'affirmant par exemple comme un mode souple de rabattement et de desserte locale, commence-t-elle tout juste à s'amorcer ? Les réseaux de surface en périphérie, trop fortement concurrencés par l'automobile et incapables de répondre à une demande de transport trop parcellisée, seraient-ils au contraire voués au déclin avant même d'avoir connu une phase de maturité ? S'il s'avérait qu'un réseau puisse disparaître après s'être trouvée en situation de complémentarité puis de concurrence avec un autre, la réintroduction des tramways, que l'autobus avait supplanté au début de ce siècle, devrait sans doute être examinée avec attention.

\section{NOTES}

1. Cet article utilise certains résultats de deux études menées pour la RATP et le Groupement de Recherches «Réseaux 》 du CNRS, dans le cadre de l'analyse de récentes transformations des réseaux d'autobus en banlieue parisienne : Offner, Sander 1990, et Legendre d'Anfray, Sander 1993.

2. L'étude de cas présentée ici est consacrée à un secteur de l'Est parisien cerné par la ligne A du RER, le chemin de fer de «Grande Ceinture » et la limite administrative de Paris (Figure 1), récemment restructuré par la RATP. Les conclusions du présent papier, bien que nourries d'une bibliographie plus générale relative à l'histoire des transports (Lagarrigue 1956 ; Daumas et al. 1977 ; Margairaz 1989), ne peuvent être étendues à l'ensemble de la banlieue parisienne qu'avec une grande prudence. Il est probable, en particulier, qu'elles ne s'appliquent pas directement à l'Ouest pari- sien, dont les principes d'urbanisation sont, dès le second Empire, différents de ceux observés à l'Est.

3. Respectivement : «Compagnie Générale des Omnibus » et « Compagnie Impériale des Voitures de Paris ». La CGO, issue de la fusion d'une dizaine de compagnies parisiennes, obtient le monopole de l'exploitation des omnibus dans Paris à partir de 1855.

4. En 1855, la Compagnie Générale des Omnibus obtient en effet le monopole de la circulation et du stationnement des omnibus, ces nouveaux véhicules à la capacité plus importante, à Paris. En banlieue, certaines lignes sont alors rachetées par cette compagnie, dans le but de maintenir à ses lignes de Paris un "précieux apport de clientèle » (Lagarrigue 1956). Les dessertes ne sont toutefois que très peu modifiées.

5. Sur le site présenté ici, deux de ces services apparaissent, de 
Montreuil et Rosny-sous-Bois vers Vincennes, en même temps qu'une seconde ligne dessert la gare de Vincennes depuis Paris, doublant celle qui se dirigeait vers le Château.

6. Lorsque de nombreux véhicules doivent se déplacer et stationner dans des quartiers constitués de voies anciennes relativement étroites et aux nombreux sens uniques, comme c'est le cas, par exemple, à Mairie de Montreuil.

7. D'après ces auteurs, « dans l'esprit des conventions de 1910, il s'agit d'assurer à chaque mode de traction une fonction spécifique dans la desserte urbaine et suburbaine : l'autobus aux petits parcours, le tramway électrique aux relations entre zones suburbaines et Paris mais également aux petits parcours intra-muros, le métro aux longs parcours circulaires ou transversaux ».

8. Ces dates sont celles retenues par L. Lagarrigue dans l'élaboration de son atlas (Lagarrigue 1956).
9. Apparition des « services rabatteurs », fermetures de certaines lignes lors de l'apparition d'une desserte ferrée.

10. Dans le but d'assurer une mission de service public. Le monopole de la STCRP s'étend, contrairement à celui de la CGO, à la banlieue.

11. Daumas et al. soulignent que suite à une demande du conseil municipal de Nanterre, la Compagnie des chemins de fer de l'Ouest répond que « le tarif ouvrier n'a été créé qu'en vue de permettre aux ouvriers d'habiter la banlieue où on peut vivre dans des conditions plus économiques ». C'est ainsi que « les parcours dans le sens Paris-banlieue ou intercommunaux ne font l'objet d'aucune tarification spéciale ».

12. Passage fréquent des véhicules aux heures « de pointe», beaucoup plus rare en heure « creuse ».

BIBLIOGRAPHIE

Arbelot G. 1973. La grande mutation des routes de France au milieu du XVII ${ }^{\mathrm{e}}$ siècle. Annales ESC 3 : 765-791.

Avramides J.M., Bessiere L., PinOn P. [n.d.] Site et développement urbain. «Groupe d'études et de recherches paysage urbain ».

Daumas M., Fontanon C., Jigaudon G., Larroque D. 1977. Analyse historique de l'évolution des transports en commun dans la région parisienne de 1855 à 1939. A.T.P. Socio-économie des Transports.

Dupuy G. (sous la direction de). 1988. Réseaux territoriaux. Paris, Paradigme.

HANNING G., 1975. La trame foncière comme organisatrice de la mise en forme du paysage. IAURIF.

LAGARRIGUE L. 1956. Cent ans de transport en commun dans la région Parisienne. 4 vol. RATP.

Legendre D'AnFray P., SANDER A. 1993. Points de réseaux et formes urbaines, une analyse de la correspondance entre autobus en région parisienne. RATP, Réseau 2000, GDR « Réseau », CNRS.

LEPETIT B. 1986 « L'impensable réseau, les routes françaises avant le chemin de fer ». Cahiers du groupe «Réseaux» 5 (juillet) : 11-29.

Margairaz M. 1989. Histoire de la RATP. Albin Michel.

Margail F., Doniol-Shaw G., Legendre D'Anfray P. 1996. La gestion du pôle Galliéni-Porte de Bagnolet. Les Annales de la Recherche Urbaine 71 (juin) : 127-136.

Menerault PH. 1991. Réseaux de transports et solidarités territoriales en milieu urbain. Thèse de doctorat, Institut d'Urbanisme de Paris XII-Val-de-Marne.

OFFNER J.-M. 1988. La disparition des tramways en France. Chemins de fer 388 (janvier-février) : 6-10.

OfFNER JJ.-M. 1992. Les déplacements urbains. Problèmes politiques et sociaux 960 (octobre), La Documentation Française.
OFFNER J.-M. 1993. Le développement des réseaux techniques : un modèle générique. Flux 13/14 (juillet-décembre) : 11-18.

OfFner J.-M, SANDER A. 1990. Les points-clés d'Autrement Bus, des théories à la pratique. Analyse de la mise en ouvre d'une innovation à la RATP. GDR « Réseaux »-RATP Réseau 2000.

Papayanis N. 1993. The Genesis of Modern Public Transportation in Paris during the First Half of the Nineteenth Century. Actes du Colloque Citadins, techniques et espaces urbains du XVIII e siècle à nos jours de La Cité des Sciences et de l'Industrie, de l'IHMC et de France-Télécom tenu à Paris les 26 et 27 janvier.

PENY A. 1990. Entre ville et réseau, la station de métro. Revue d'Histoire des Chemins de Fer 2 (printemps).

PICON A. 1992. L'invention de l'ingénieur moderne, L'école des Ponts et Chaussées 1747-1851. Paris, Presses de l'ENPC.

SCHERRER F. 1992. L'égout patrimoine urbain. L'évolution sur la longue durée du réseau d'assainissement de Lyon. Thèse de doctorat, Institut d'Urbanisme de Paris XII-Val-de-Marne.

\section{Sources iconographiques}

Carte des tramways du département de la Seine, 1867, BHVP, G541.

Autobus, tramways, bateaux, lignes de banlieue, Société des Transports en Commun de la Région Parisienne, 1931, BHVP, $8^{\circ}$ AT 111.

Carte des Autobus de la banlieue parisienne, 1937, BHVP, G 1024 .

Autobus Paris Banlieue, Ed. Chaix, 1946, BVVP, $8^{\circ}$ AT 115.

Plan des autobus de banlieue, 1964, BHVP, G 1071.

Plan des autobus de la banlieue parisienne, 1972, BHVP, G1056.

Plan des autobus Est Parisien, RATP, 1993. 